\title{
Using electron vortex beams to determine chirality of crystals in transmission electron microscopy
}

\author{
Roeland Juchtmans, Armand Béché, Artem Abakumov, Maria Batuk, and Jo Verbeeck \\ EMAT, University of Antwerp, Groenenborgerlaan 171, 2020 Antwerp, Belgium \\ (Received 8 October 2014; revised manuscript received 5 February 2015; published 26 March 2015)
}

\begin{abstract}
We investigate electron vortex beams elastically scattered on chiral crystals. After deriving a general expression for the scattering amplitude of a vortex electron, we study its diffraction on point scatterers arranged on a helix. We derive a relation between the handedness of the helix and the topological charge of the electron vortex on one hand and the symmetry of the higher-order Laue zones in the diffraction pattern on the other for kinematically and dynamically scattered electrons. We then extend this to atoms arranged on a helix as found in crystals which belong to chiral space groups and propose a method to determine the handedness of such crystals by looking at the symmetry of the diffraction pattern. In contrast to alternative methods, our technique does not require multiple scattering, which makes it possible to also investigate extremely thin samples in which multiple scattering is suppressed. In order to verify the model, elastic scattering simulations are performed, and an experimental demonstration on $\mathrm{Mn}_{2} \mathrm{Sb}_{2} \mathrm{O}_{7}$ is given in which we find the sample to belong to the right-handed variant of its enantiomorphic pair. This demonstrates the usefulness of electron vortex beams to reveal the chirality of crystals in a transmission electron microscope and provides the required theoretical basis for further developments in this field.
\end{abstract}

DOI: 10.1103/PhysRevB.91.094112

PACS number(s): 61.05.J-, 03.65.Vf, 41.85.-p

\section{INTRODUCTION}

An object is said to be chiral if it is not superposable onto its mirror image by rotating and/or translating it. As such, chiral objects come in two forms, an original and its mirror image, called enantiomorphs. In crystals, chirality manifests itself in the crystal's space groups (SGs). These can be divided in three classes, the first of which contains all the SGs with an improper symmetry operation (inversion center, mirror plane, glide plane, or rotoinversion axes), which represent nonchiral crystals. Class II includes the 22 SGs which have a screw axis apart from the $2_{1}$ screw axis. These SGs are chiral and can be divided into 11 enantiomorphic pairs, which are listed in Table I. Because of the screw axis, atoms in these crystals are arranged in a helical way. The last class contains SGs which possess only proper rotations and/or a 21 screw axis. Crystals belonging to these space groups also are chiral, but when mirrored, the space group of the crystal does not change; therefore the SG itself is achiral.

Determining the chirality of a crystal is important when investigating its chiral properties, e.g., optical activity, chiral dichroism, and chemical reactions with other chiral molecules. Doing this in a transmission electron microscope, however, appears to be a difficult task. In transmission electron microscopy, the crystal is seen [at least for the so-called zeroth-order Laue zone (ZOLZ) in the diffraction pattern] as a two-dimensional projection through the thickness of the sample. Because of this, one can never distinguish between enantiomorphs since mirroring the crystal along the projection plane changes the handedness of the material but not its projection on this plane. Therefore, all three dimensions have to be taken into account. In a technique proposed by Goodman and Secomb [1], the chirality is recovered by rotating the object and taking several ZOLZ electron diffraction patterns. Another way to gather information on the dimension perpendicular to the projection plane is to look at so-called higher-order Laue zones (HOLZs) [2]. Friedel's law, however, imposes extra symmetry in the diffraction pattern that does not allow us to determine the chirality in the kinematical approximation [3]. Therefore multiple scattering (dynamical approximation) is used to break this symmetry and to allow us to determine the chirality. All methods developed so far thus require dynamical simulations and depend in a sensitive way on sample thickness. An additional difficulty appears as not all HOLZ diffraction spots are sensitive to the handedness of the crystal, and one has to identify them to be able to determine the chirality [4,5]. In this work, we propose the use of electron vortex beams to break the symmetry imposed by Friedel's law as an alternative way to distinguish SGs in one of the enantiomorphic SG pairs from Table I without having to rely on dynamical scattering.

As described theoretically by Nye and Berry [6], vortex waves are solutions of the three-dimensional (3D) wave equation with an angular-dependent phase factor of the form

$$
\Psi(\boldsymbol{r})_{m}=\psi(r, z) e^{i m \phi},
$$

with $r$ and $\phi$ being the radial and azimuthal coordinates with respect to the wave propagation axis $z$. The number $m$ is referred to as the topological charge. As an eigenstate of the angular momentum operator $L_{z}=-i \hbar \frac{\partial}{\partial \phi}$, vortex waves carry a well-defined angular momentum of $m \hbar$ per photon or electron [7]. In the center of the vortex, the phase is ill defined, and the intensity of the beam becomes zero due to destructive interference, resulting in the typical donut-like intensity profile. Ever since the first experimental realization of optical vortex beams over two decades ago [8], they have been the subject of active research, which has led to applications varying from nanomanipulation [9-11] to astrophysics [12-15] to telecommunication [16-18]. More recently, the first electron vortex beam [19] was created by making a phase plate of stacked graphite layers [20], followed by holographic reconstruction as a more reliable way of producing electron vortices [21]. New ways are still being developed, and currently, single-mode vortex beams at high current as well as atomic-size vortices have been 
TABLE I. The 22 chiral space groups in class II, divided into 11 enantiomorphic space-group pairs.

\begin{tabular}{cc}
\hline \hline & Enantiomorphic space-group pairs \\
\hline 1 & $\left(P 4_{1}, P 4_{3}\right)$ \\
2 & $\left(P 4_{1} 32, P 4_{3} 32\right)$ \\
3 & $\left(P 4_{1} 22, P 4_{3} 22\right)$ \\
4 & $\left(P 4_{1} 22, P 4_{3} 2\right)$ \\
5 & $\left(P 6_{1}, P 6_{5}\right)$ \\
6 & $\left(P 6_{2}, P 6_{4}\right)$ \\
7 & $\left(P 6_{1} 22, P 6_{5} 22\right)$ \\
8 & $\left(P 6_{2} 22, P 6_{4} 22\right)$ \\
9 & $\left(P 3_{1}, P 3_{2}\right)$ \\
10 & $\left(P 3_{1} 12, P 3_{2} 12\right)$ \\
11 & $\left(P 3_{1} 21, P 3_{2} 21\right)$ \\
\hline \hline
\end{tabular}

realized [22,23]. Several studies suggest practical use of electron vortex beams in electron energy-loss magnetic chiral dichroism experiments [21], nanomanipulation [24], spinpolarization devices [25], and magnetic plasmons [26].

As Friedel's law is applicable only to plane-wave scattering, diffraction patterns obtained from vortex beams can be fundamentally different when scattered on enantiomorphic objects, even in the kinematical approximation. The central question in this paper is then, Are electron vortex beams capable of distinguishing chiral crystals within the kinematical approximation? We will tackle this problem by looking at crystals in chiral space groups in which the atoms are arranged along a helical axis.

\section{THEORETICAL FORMULATION}

\section{A. Vortex scattering in cylindrical coordinates within the kinematical approximation}

Assuming the scattered part of the wave to be much smaller than its incoming part, the first-order Born approximation considers the potential a small perturbation on free space and describes only single-scattering events. Within this approximation the scattering amplitude for an incoming wave $\psi_{0}(\boldsymbol{r})$ to scatter on a potential $V(\boldsymbol{r})$ to a plane wave with wave vector $\boldsymbol{k}^{\prime}$ is proportional to [27]

$$
A\left(\boldsymbol{k}^{\prime}\right)=\left\langle\boldsymbol{k}^{\prime}|V(\boldsymbol{r})| \psi_{0}\right\rangle \propto \int d \boldsymbol{r} e^{-i \boldsymbol{k}^{\prime} \cdot \boldsymbol{r}} V(\boldsymbol{r}) \psi_{0}(\boldsymbol{r}) .
$$

Our aim is to find an expression for the vortex beam scattering amplitude for which $\psi_{0}(\boldsymbol{r})=\psi(r) e^{i m \phi} e^{i k_{z} z}$. Starting from Eq. (3), we get for a vortex electron with topological charge $m$ on a potential $V(\boldsymbol{r})$

$$
A_{m}\left(\boldsymbol{k}^{\prime}\right)=\int d \boldsymbol{r} e^{-i \boldsymbol{k}^{\prime} \cdot \boldsymbol{r}} V(\boldsymbol{r}) e^{i k_{z} z} e^{i m \phi} \psi(r) .
$$

Since vortex beams are most easily described in cylindrical coordinates, it will be convenient to expand the potential in a cylindrical symmetric basis of Bessel functions [28].

$$
\begin{aligned}
V(\boldsymbol{r})= & \frac{1}{\sqrt{2 \pi}} \int d k_{z}^{\prime \prime} \int_{0}^{\infty} d k_{\perp}^{\prime \prime} k_{\perp}^{\prime \prime} \\
& \times \sum_{m^{\prime \prime}=-\infty}^{\infty} V_{m^{\prime \prime}}\left(k_{\perp}^{\prime \prime}, k_{z}^{\prime \prime}\right) J_{m}^{\prime \prime}\left(k_{\perp}^{\prime \prime} r\right) e^{i m^{\prime \prime} \phi} e^{i k_{z}^{\prime \prime} z},
\end{aligned}
$$

with

$$
\begin{aligned}
V_{m^{\prime \prime}}\left(k_{\perp}^{\prime \prime}, k_{z}^{\prime \prime}\right)= & \frac{1}{\sqrt{2 \pi}} \int d z \int_{0}^{\infty} d r \int_{0}^{2 \pi} d \phi \\
& \times r V(r, \phi, z) J_{m^{\prime \prime}}\left(k_{\perp}^{\prime \prime} r\right) e^{-i m^{\prime \prime} \phi} e^{-i k_{z}^{\prime \prime} z} .
\end{aligned}
$$

Using these equations, we can write the scattering amplitude as

$$
\begin{aligned}
A_{m}\left(\boldsymbol{k}^{\prime}\right)= & \int_{0}^{\infty} d k_{\perp}^{\prime \prime} k_{\perp}^{\prime \prime} \sum_{m^{\prime}=-\infty}^{\infty}(-i)^{m^{\prime}} V_{m^{\prime}-m}\left(k_{\perp}^{\prime \prime}, k_{z}^{\prime}-k_{z}\right) \\
& \times e^{i m^{\prime} \phi_{k^{\prime}}} \int_{0}^{\infty} d r r J_{m^{\prime}-m}\left(k_{\perp}^{\prime \prime} r\right) J_{m^{\prime}}\left(k_{\perp}^{\prime} r\right) \psi(r) .
\end{aligned}
$$

Equation (6) is the most general expression to describe vortex scattering in cylindrical coordinates on a potential determined by its polar expansion coefficients $V_{m^{\prime \prime}}\left(k_{\perp}^{\prime \prime}, k_{z}^{\prime \prime}\right)$.

\section{B. Vortex scattering on a helix}

The potential of a helical distribution of point scatterers on a helix with pitch $P$, radius $R$, and $Q$ point scatterers on each period of the helix can be written as

$$
\begin{aligned}
V(\boldsymbol{r})= & \sum_{j, n=-\infty}^{\infty} \delta(r-R) \\
& \times \delta\left(\phi-a \frac{2 \pi z}{P}+2 \pi n\right) \delta\left(z-\frac{j}{Q} P\right),
\end{aligned}
$$

where $a=+1$ in the case of a right-handed helix and $a=-1$ for a left-handed helix. Starting from Eq. (5), we find the potential's expansion coefficients:

$$
V_{m, v}\left(q_{\perp}\right)=\frac{R Q}{P} J_{m}\left(q_{\perp} R\right) \sum_{N} \delta\left(\frac{a m+v}{Q}-N\right),
$$

where $v \in \mathbb{Z}$ and $q_{z}=v \frac{2 \pi}{P}$ is quantized because of the periodicity of the potential in the $z$ direction.

When dropping the constant $\frac{R Q}{P}$, filling the expansion coefficients (8) into Eq. (6) and using some simple algebra given in Appendix $B$ give us the scattering amplitude

$$
\begin{aligned}
A_{m}\left(\boldsymbol{k}^{\prime}\right)= & e^{i(m-a v) \phi_{k^{\prime}}} \sum_{N=-\infty}^{\infty}(-i)^{N Q} e^{i N Q \phi_{k^{\prime}}} \\
& \times J_{m-a v+N Q}\left(k_{\perp}^{\prime} R\right) \psi(R) .
\end{aligned}
$$

Note that because of the periodicity in the $z$ direction, the transferred forward momentum, $k_{z}-k_{z}^{\prime}=v \frac{2 \pi}{P}$, is quantized. This means the diffraction pattern consists of discrete rings which coincide with higher-order Laue zones in conventional electron-beam diffraction.

When looking at the scattering amplitude for certain rings for which $v=a m+n Q$ or, equivalently, $m-a v=n Q$, $n \in \mathbb{Z}$, we get

$$
\begin{aligned}
& \left.A_{m}\left(k_{\perp}^{\prime}, \phi_{k^{\prime}}, k_{z}^{\prime}\right)\right|_{m-a v=n Q} \\
& =J_{0}\left(k_{\perp}^{\prime} R\right)+\sum_{N=1}^{\infty} 2(-i)^{N Q} J_{N Q}\left(k_{\perp}^{\prime} R\right) \cos \left(N Q \phi_{k^{\prime}}\right),
\end{aligned}
$$


for which

$$
\left.A_{m}\left(k_{\perp}^{\prime}, \phi_{k^{\prime}}, k_{z}^{\prime}\right)\right|_{m-a v=n Q}=\left.A_{m}^{*}\left(k_{\perp}^{\prime}, \phi_{k^{\prime}}, k_{z}^{\prime}\right)\right|_{m-a v=n Q} .
$$

The intensity of the diffraction pattern $I\left(\boldsymbol{k}^{\prime}\right)$ is given by the amplitude squared $\left|A\left(\boldsymbol{k}^{\prime}\right)\right|^{2}$, and Eq. (10) shows these diffraction rings will be centrosymmetric. Equation (11) is the most interesting result in this paper. It means that the chirality of the helix can be seen from the symmetry of the HOLZs, at least for helices with odd-screw-axis symmetry. Take, for instance, a right- or left-handed threefold screw axis for which $a=+1$ or $a=-1$, respectively, and $Q=3$. When we look at the first-order Laue zone (FOLZ; $v=1$ ) using a vortex beam with topological charge $m=1$, we see that the relation $1=v=a m+n Q=a 1+n 3$ is only valid for $n=0$ and $a=1$. Thus the FOLZ of the right-handed helix will possess centrosymmetry, while that of the left-handed helix will not.

\section{Effect of multiple scattering on the symmetry of HOLZ}

The previous derivation of the symmetry of the diffraction rings was done in the kinematical approximation. However, electrons generally are subject to multiple scattering even when scattered on extremely thin samples. In this section we extend our findings on the symmetry of the FOLZ in the kinematical approximation to the situation where the electron is dynamically scattered.

Remember from the previous section that the scattering amplitude of a vortex electron with topological charge $m$ to scatter on a crystal to a plane wave with wave vector $\boldsymbol{k}$ is given by

$$
\begin{aligned}
A(\boldsymbol{k}) & =\left\langle\boldsymbol{k}|V(\boldsymbol{r})| \psi_{m}\right\rangle=\int d \boldsymbol{r} e^{-i \boldsymbol{k} \cdot \boldsymbol{r}} V(\boldsymbol{r}) \psi_{m}(\boldsymbol{r}) \\
& =\mathcal{F}\left[V(\boldsymbol{r}) \psi_{m}(\boldsymbol{r})\right](-\boldsymbol{k})=\mathcal{F}[V] * \mathcal{F}\left[\psi_{m}\right](-\boldsymbol{k}),
\end{aligned}
$$

where the asterisk denotes a convolution product.

Let us now consider an electron that is subject to two scattering events while interacting with the potential. The scattering amplitude for the electron to scatter to a wave with wave vector $\boldsymbol{k}$ now is given by

$$
A_{m}^{(2)}(\boldsymbol{k})=\int d \boldsymbol{k}^{\prime}\left\langle\boldsymbol{k}|V(\boldsymbol{r})| \boldsymbol{k}^{\prime}\right\rangle\left\langle\boldsymbol{k}^{\prime}|V(\boldsymbol{r})| \psi_{m}\right\rangle .
$$

It describes a scattering event of an electron in vortex state $\left|\psi_{m}\right\rangle$ to scatter to any plane wave $\left|\boldsymbol{k}^{\prime}\right\rangle$ which is then scattered a second time to the final state $|\boldsymbol{k}\rangle$. We can rewrite this as

$$
\begin{aligned}
A_{m}^{(2)}(\boldsymbol{k}) & =\int d \boldsymbol{k}^{\prime} \mathcal{F}[V]\left(\boldsymbol{k}^{\prime}-\boldsymbol{k}\right)\left\{\mathcal{F}[V] * \mathcal{F}\left[\psi_{m}\right]\left(-\boldsymbol{k}^{\prime}\right)\right\} \\
& =\mathcal{F}[V] * \mathcal{F}[V] * \mathcal{F}\left[\psi_{m}\right](-\boldsymbol{k}) .
\end{aligned}
$$

When only considering scattering to and from the ZOLZ and FOLZ, Eq. (14) includes ZOLZ-ZOLZ, ZOLZ-FOLZ, FOLZ-ZOLZ. and FOLZ-FOLZ scattering. The latter contains two high-angle scattering events between two different Laue zones. and its contribution will be significantly smaller than that of the other events, which are schematically drawn in Fig. 1. Neglecting these, we show in Appendix $\mathrm{C}$ that double scattering preserves the centrosymmetry we found before.

$$
\left.A_{m}^{(2)}\left(k_{\perp}, \phi_{k}\right)\right|_{k \in F O L Z}=\left.A_{m}^{*(2)}\left(k_{\perp}, \phi_{k^{\prime}}+\pi\right)\right|_{k \in F O L Z} .
$$

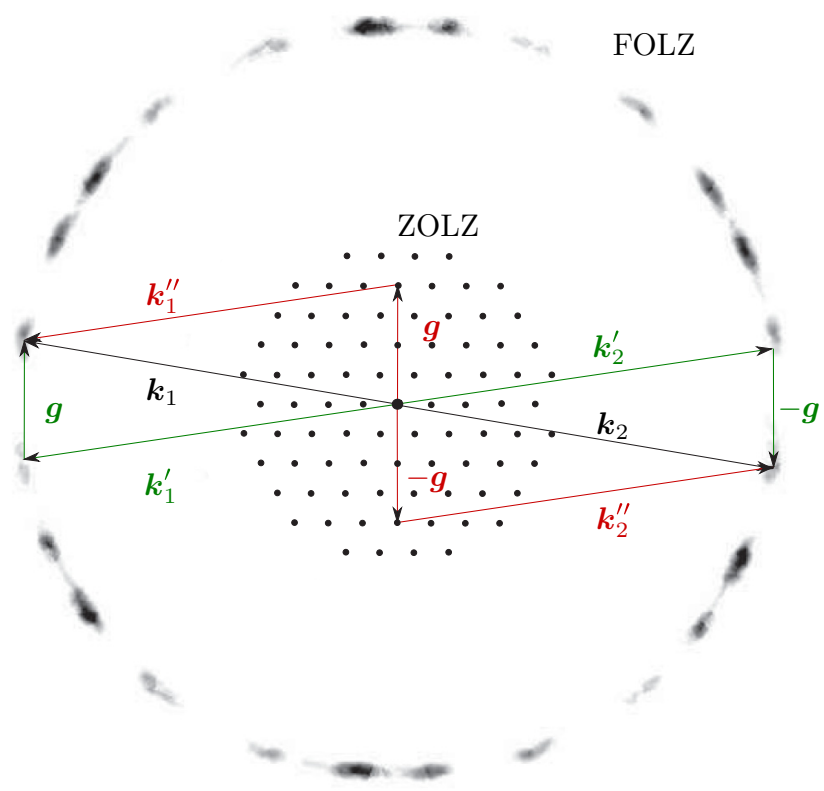

FIG. 1. (Color online) Schematic diagram of the scattering paths of a focused vortex beam to scatter to points $\boldsymbol{k}_{1}$ and $\boldsymbol{k}_{2}$ in the FOLZ opposite each other. The black path represents the first-order Born approximation in which the electron scatters only once. The red path is a second-order Born approximation in which the electron first scatters to a point in the ZOLZ and then to the FOLZ. The green path represents an event in which the electron scatters to the FOLZ and then scatters within this zone through a scattering vector of the ZOLZ. When neglecting multiple scattering between different Laue zones, the symmetry of the diffraction pattern is also conserved in the dynamical approximation.

Moreover, this can easily be extended to dynamical scattering up to any order when neglecting the scattering paths with more than one inter-Laue zone scattering event. We should note, however, that when more scattering events are taken into account, the relative contribution of scattering paths with more than one inter-Laue zone scattering event will increase, eventually breaking the symmetry of the FOLZ when looking at very thick samples.

\section{ELECTRON VORTEX BEAMS IN A TEM}

We apply the model of point scatterers on a helix as an approximation of a focused vortex beam scattered on a helical crystal when the vortex probe is centered on a screw axis and predominantly illuminates a set of heavy atoms distributed on a helix as sketched in Fig. 2. The crystal potential in real space can then be approximated by the convolution of the helix potential with the potential of a single heavy atom, while in Fourier space it is given by the product of the Fourier transform of the helix and the atomic potential. Since the latter is spherically symmetric, the angular dependency of the total Fourier transform stays the same. This means that, if we neglect the phase and intensity variation of the vortex beam over the size of one atom, the intensity profile of each Laue zone will still be given by Eq. (9). The only effect caused by the atomic scattering factor is a modulation of this intensity with respect to the scattering angle. 


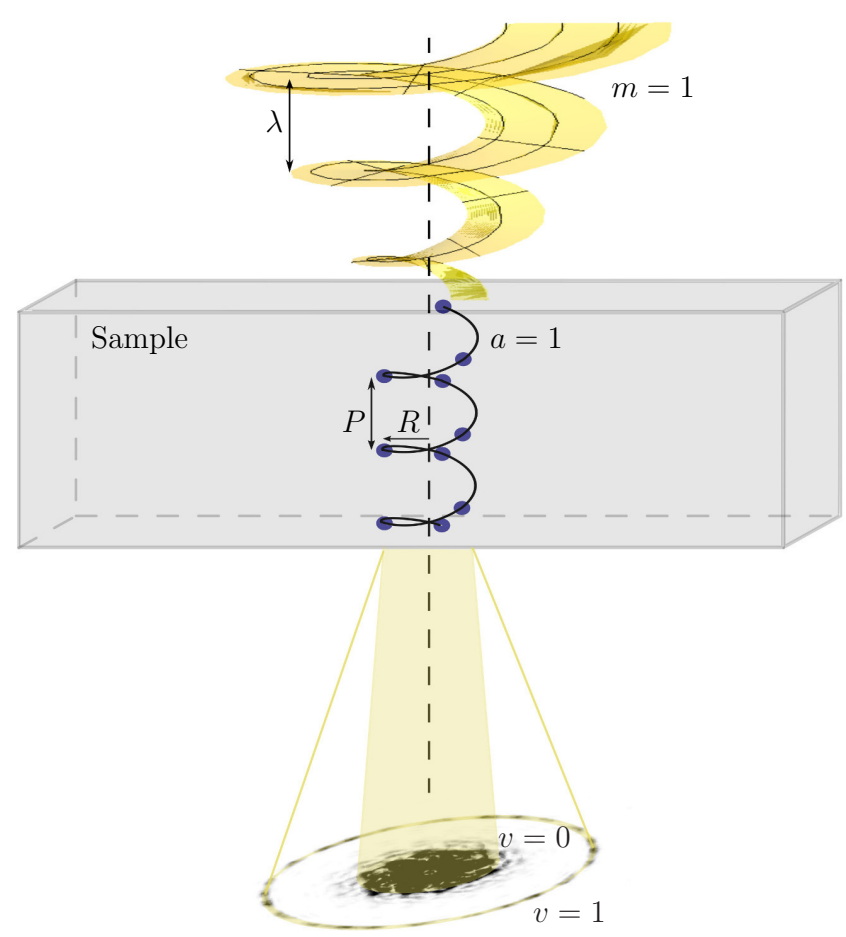

FIG. 2. (Color online) Schematic representation of the experimental setup. A vortex beam is focused directly on a screw axis in the sample further simplified assuming elastic scattering is dominated by the helically arranged heavy atoms. The radius of the vortex is chosen such that it matches the distance of the atoms to the screw axis.

Equation (9) shows that vortex beam diffraction is sensitive to the chirality of crystals. When a vortex beam is focused on a screw axis with one heavy atom nearby, the HOLZs will look different for enantiomorphs. Moreover, for crystals with a threefold screw axis ( $Q=3$ ), some of the HOLZs will become centrosymmetric depending on the topological charge of the vortex and the chirality of the crystal, revealing the latter at first glance. In what follows we will demonstrate our approach on $\mathrm{Mn}_{2} \mathrm{Sb}_{2} \mathrm{O}_{7}$. From Scott et al. [29] we know this crystal belongs to space group $P 3_{1} 21$ or $P 3_{2} 21$, in which the atoms lie on a right- or left-handed threefold helix, respectively. One of the three inequivalent screw axes only has one heavy $S b$ atom in its vicinity, and electron scattering of a focused (vortex) electron beam centered on this axis approximates the situation of scatterers on a helix. We will determine the chirality of the SG by determining the handedness of this screw axis, which we will refer to without further specification.

In order to verify whether the symmetry predicted in Eq. (11) still holds for a vortex beam scattered on a real crystal (i.e., not only a helical arrangement of atoms) we perform multislice simulations using the program STEMSIM [30] for which we include full dynamical scattering and spreading of the beam. We look at $\mathrm{Mn}_{2} \mathrm{Sb}_{2} \mathrm{O}_{7}$ along the crystallographic [001] direction, the direction of the screw axis, and focus the vortex beam over it. We choose the semiconvergence angle of the beam such that the radius of the vortex matches the distance of the $\mathrm{Sb}$ atoms to the screw axis, i.e., $1.2 \AA$. For a $300 \mathrm{keV}$ vortex beam with $m= \pm 1$ the convergence angle corresponding to this criterion is $8 \mathrm{mrad}$. The resulting
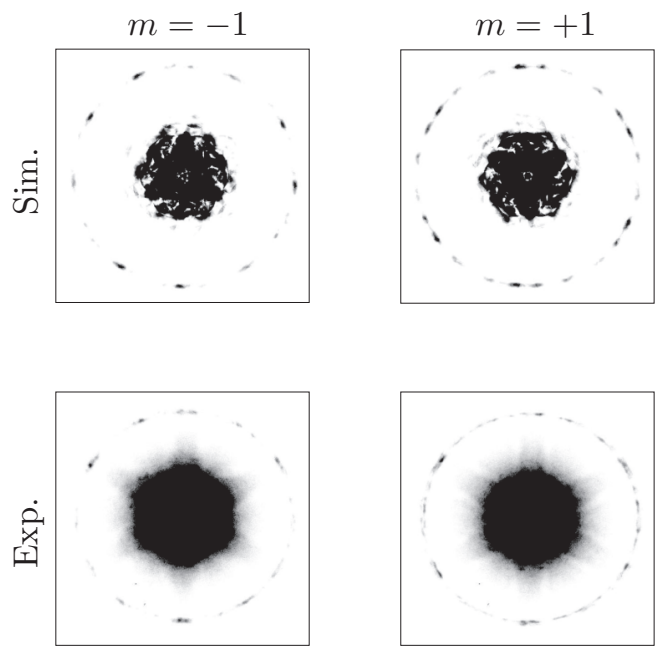

FIG. 3. (top) Multislice simulation of the first-order Laue zone of the diffraction pattern of (left) an $m=-1$ vortex and (right) an $m=$ +1 vortex scattered on right-handed $\mathrm{Mn}_{2} \mathrm{Sb}_{2} \mathrm{O}_{7}$, with $\mathrm{SG} P 3_{1} 21$. The vortex is centered along the screw axis with the size of the vortex equal to the distance of the $\mathrm{Sb}$ atoms from the screw axis, approximately $1.2 \AA$. The energy of the probe is $300 \mathrm{keV}$, the convergence angle is $8 \mathrm{mrad}$, and the spherical aberration is $1 \mu \mathrm{m}$. The thickness of the sample is $20 \mathrm{~nm}$. The centrosymmetry of the first-order ring can be seen for the right-handed enantiomorph for $m=+1$. (bottom) Experimental CBED pattern of a $1.2-\AA$-sized vortex probe pointed at a threefold screw axis in $\mathrm{Mn}_{2} \mathrm{Sb}_{2} \mathrm{O}_{7}$ for $m=-1$ (left) and $m=$ +1 (right). The contrast was adapted to show only the peaks in the FOLZ.

convergent beam electron diffraction (CBED) patterns for left- and right-handed vortices scattered on a right-handed 20-nm-thick $\mathrm{Mn}_{2} \mathrm{Sb}_{2} \mathrm{O}_{7}$ sample are given in Fig. 3. Here we see that the FOLZ of the left-handed vortex shows only threefold symmetry, whereas in the case of the right-handed vortex, the FOLZ shows sixfold symmetry.

Next, we demonstrate our technique experimentally on crushed $\mathrm{Mn}_{2} \mathrm{Sb}_{2} \mathrm{O}_{7}$ crystals deposited on a carbon grid. QuAnTem, a double $C_{s}$ corrected FEI Titan ${ }^{3}$ microscope, operating at $300 \mathrm{keV}$, was used to record the experimental diffraction patterns. The vortex beams were created with an aperture on which a magnetized needle is mounted, similar to the setup described by Béché et al. [22].

After making a high-resolution image of a nanocrystal in the [001] zone axis, the beam was pointed exactly on the screw axis, and the diffraction pattern was recorded with a CCD camera (1-s exposure time).

We recorded five diffraction patterns using a vortex with topological charge $m \approx+1$ and six patterns with $m \approx-1$ scattered on the same crystal. Because the beam is much smaller than the unit-cell size, the symmetry of the diffraction pattern is very sensitive to the position of the probe. Two images recorded with opposite vortices which showed the highest threefold symmetry in the diffraction pattern are compared with the simulated ones in Fig. 3. We adjusted the contrast such that only the peaks in the FOLZ are visible and see that the recorded FOLZs qualitatively match the simulations for the right-handed crystal rather well. 

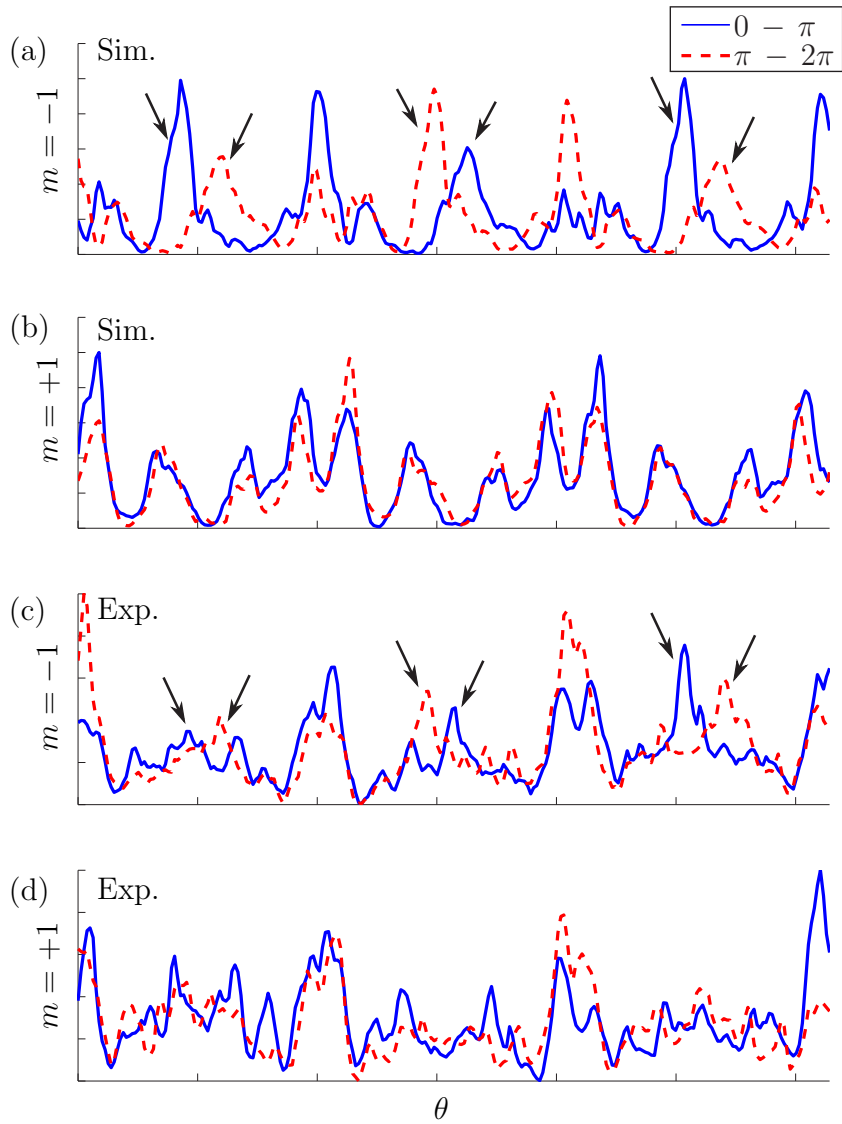

FIG. 4. (Color online) Comparison of the (a) and (b) simulated and (c) and (d) experimental $I_{F O L Z}(\theta)$ for $\theta \in\{0, \pi\}$ (blue) and $\theta \in$ $\{\pi, 2 \pi\}$ (red) for (a) and (c) $m=-1$ and (b) and (d) $m=+1$ on the $P 3_{1} 21$ enantiomorph of $\mathrm{Mn}_{2} \mathrm{Sb}_{2} \mathrm{O}_{7}$. From the position of the peaks it is clear that the $m=+1$ vortex scattered on the right-handed screw axis gives a centrosymmetric FOLZ, which is not the case for the $m=-1$ vortex, where the differences are indicated by arrows.

As it is our intention to determine the chirality of the crystal without comparing the experiment with simulation, we look at the symmetry of the FOLZ ring. To do so in more detail, we plot $I_{F O L Z}(\theta)$, the azimuthal intensity profile of the FOLZ integrated over a small radius $(r, r+d r)$, in Fig. 4 for the azimuthal coordinate $\theta \in\{0, \pi\}$ (in blue) and for $\theta \in\{\pi, 2 \pi\}$ (in red). Although there are some differences between the $(0, \pi)$ and $(\pi, 2 \pi)$ ranges for the simulated $m=+1$, the position of the peaks is clearly centrosymmetric, which is not at all the case for the simulated $m=-1$ (see arrows in Fig. 4). When looking at the experimental $I_{F O L Z}(\theta)$, it can be seen that most peaks in the $m=+1$ data have a peak at $\theta^{\prime}=\theta+\pi$. For $m=-1$ we can see peaks in the $(0, \pi)$ range which are not present in $(\pi, 2 \pi)$ range, indicating that the FOLZ here is not centrosymmetric. From this we conclude that the crystal under investigation has a right-handed screw axis and thus belongs to $P 3_{1} 21$

The major difficulty of this method, and the main reason for the deviations of the symmetry in the experiment, is the probe positioning, which has to be maintained during the acquisition of the diffraction pattern.
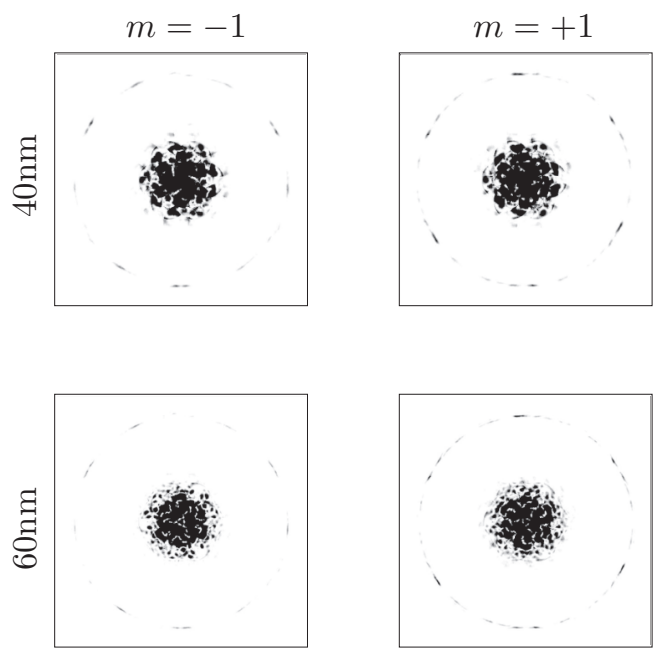

FIG. 5. Multislice simulation of the first-order Laue zone of the diffraction pattern of (left) an $m=-1$ vortex and (right) an $m=+1$ vortex scattered on right-handed $\mathrm{Mn}_{2} \mathrm{Sb}_{2} \mathrm{O}_{7}$, with thicknesses of (top) $40 \mathrm{~nm}$ and (bottom) $60 \mathrm{~nm}$. Although dynamical scattering is expected to be dominant, the centrosymmetry of the FOLZ still can be seen for $m=+1$, as was the case for the 20 -nm-thick sample.

To demonstrate the robustness of the symmetry to multiple scattering we perform multislice simulations on sample thicknesses of 40 and $60 \mathrm{~nm}$, shown in Fig. 5, where the dynamical scattering is expected to be dominant. When looking directly at the diffraction patterns, the centrosymmetry of the FOLZ of the $m=+1$ vortex appears to be conserved when scattered on the right-handed crystal, even for a sample thickness up to $60 \mathrm{~nm}$. To look at this in more detail, we compare the intensity profile of the FOLZ for $\theta \in\{0, \pi\}$ with that for $\theta \in\{\pi, 2 \pi\}$ (see Fig. 6). We can see that, although the shapes of opposite peaks are different, centrosymmetry is again present in the position of the peaks.

\section{CONCLUSION}

We showed that vortex beam diffraction can be sensitive to the chirality of a crystal, even within the kinematical approximation. As an example, we calculated analytically the scattering amplitude of a vortex scattered kinematically on point scatterers arranged on a helix and found that the angular intensity profiles of the HOLZs depend on the topological charge of the vortex and the chirality of the helix. For helices with odd-screw-axis symmetry it was found that the chirality can be determined unambiguously from the symmetry in the diffraction pattern. We extend this property to dynamically scattered electrons that scatter only once from the ZOLZ to the FOLZ, increasing the range of its applicability to more conventional sample thicknesses in the TEM.

Based on these results, we demonstrated a technique to determine the handedness of a chiral SG by focusing a vortex probe over a screw axis in the crystal. We verified the symmetry of the HOLZ with multislice simulations on $\mathrm{Mn}_{2} \mathrm{Sb}_{2} \mathrm{O}_{7}$ and gave an experimental demonstration of the technique. The major difficulty is the required accuracy of the probe positioning, which, in combination with sample drift, makes acquisition 

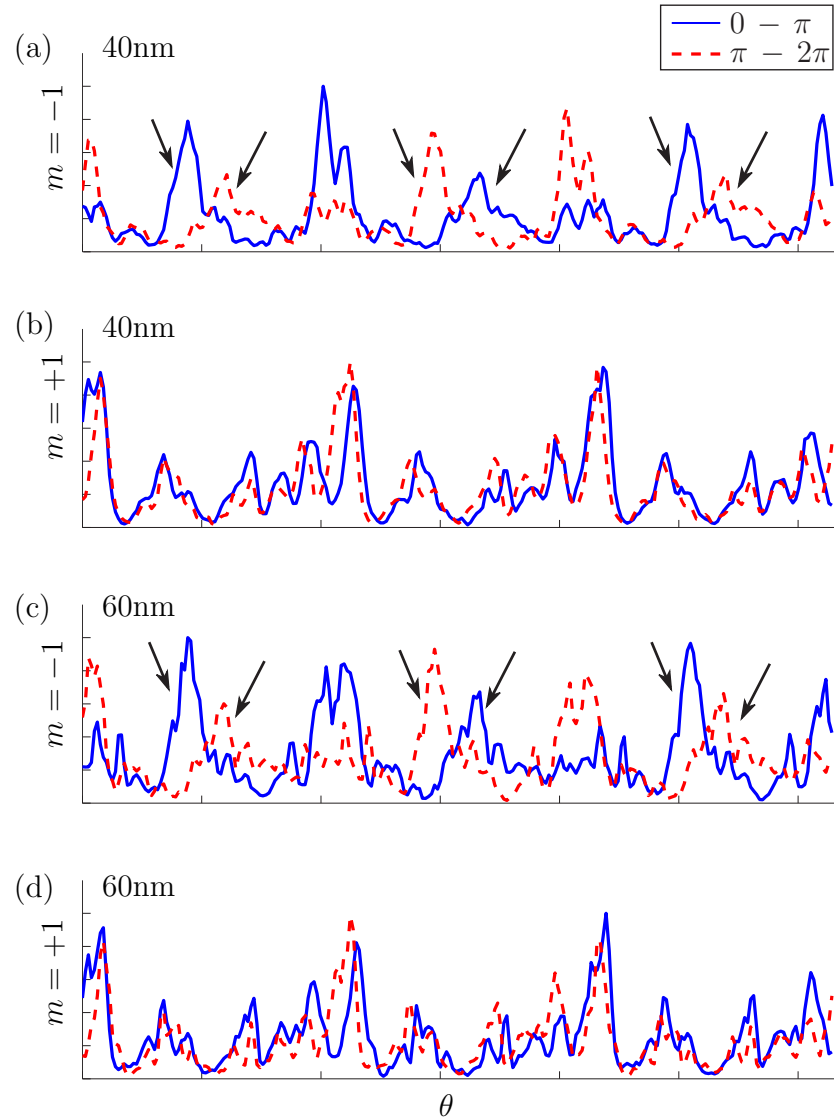

FIG. 6. (Color online) Comparison of the simulated $I_{F O L Z}(\theta)$ for $\theta \in\{0, \pi\}$ (blue) and $\theta \in\{\pi, 2 \pi\}$ (red) for (a) and (c) $m=-1$ and (b) and (d) $m=+1$ on the $P 3_{1} 21$ enantiomorph of $\mathrm{Mn}_{2} \mathrm{Sb}_{2} \mathrm{O}_{7}$ for sample thicknesses of (a) and (b) $40 \mathrm{~nm}$ and (c) and (d) $60 \mathrm{~nm}$. In (b) and (d) it can be seen that the positioning of the peaks is still centrosymmetric for samples up to $60 \mathrm{~nm}$, although the shape of the peaks gets altered. This symmetry is not at all present in the $m=-1$ vortex case, for which the main differences are indicated by arrows.

of the data cumbersome. The four-dimensional scanning transmission electron microscopy (4D-STEM) method proposed by Ophus et al. [31] might help in overcoming this problem. In this method a STEM image is recorded while taking a diffraction pattern at every scan point, thus avoiding the need to point the probe at the exact location manually.

Naturally, the use of vortices to determine the chirality of a crystal is not restricted to crystals with a screw axis with only one heavy atom as the nearest neighbor. In Appendix D we show that, in general, vortex wave diffraction is sensitive to the position of atoms in the direction perpendicular to the zone axis within the kinematical approximation, making vortex beams a potentially valuable tool to determine the chirality of crystals, even when they do not belong to one of the chiral SGs in Table I. The symmetry of the diffraction pattern, however, can no longer be used, and simulations are needed to link the experiment with the chirality of the structure.

Although this technique has its experimental challenges, it has the advantage that the chirality of a crystal can be determined solely by looking at the symmetry of the diffraction pattern, thus avoiding the need for dynamical calculations. Also, this can be done within the kinematical regime such that the chirality of very thin samples in which multiple scattering is limited can be measured. On top of that, this can be done on a local basis for each position in the crystal, opening the route for probing the chirality of crystals at atomic resolution or for investigating screw dislocations in nonchiral crystals. This work shows applications of electron vortex beam diffraction in crystallography in which the chiral character of the probe has significant influence on the diffraction pattern and its symmetry. It gives fundamental insights into how electron vortices interact with the symmetry of crystal and provides a basis for derived methods and techniques to be developed. Finally, it gives an example of the more general idea of optimizing the beam (both intensity and phase) to study specific properties, as suggested by Guzzinati et al. [32] and Rusz et al. [33].

\section{ACKNOWLEDGMENTS}

The authors acknowledge support from the FWO (Aspirant Fonds Wetenschappelijk Onderzoek-Vlaanderen); the EU under the Seventh Framework Program (FP7) under a contract for an Integrated Infrastructure Initiative, Reference No. 312483-ESTEEM2; and the European Research Council under the FP7 and ERC Starting Grant No. 278510 VORTEX.

\section{APPENDIX A: DERIVATION OF THE VORTEX SCATTERING AMPLITUDE IN CYLINDRICAL COORDINATES}

Starting from the scattering amplitude within the first-order Born approximation for a vortex electron [Eq. (3)] and writing the potential with its polar expansion coefficients [Eq. (4)], we get

$$
A_{m}\left(\boldsymbol{k}^{\prime}\right)=\frac{1}{\sqrt{2 \pi}} \int d \boldsymbol{r} \int d k_{z}^{\prime \prime} \int_{0}^{\infty} d k_{\perp}^{\prime \prime} k_{\perp}^{\prime \prime} \sum_{m^{\prime \prime}=-\infty}^{\infty} V_{m^{\prime \prime}}\left(k_{\perp}^{\prime \prime}, k_{z}^{\prime \prime}\right) J_{m^{\prime \prime}}\left(k_{\perp}^{\prime \prime} r\right) e^{i m^{\prime \prime} \phi} e^{i k_{z}^{\prime \prime} z} e^{i\left(k_{z}-k_{z}^{\prime}\right) z} e^{i m \phi} e^{-i \boldsymbol{k}_{\perp}^{\prime} \cdot \boldsymbol{r}_{\perp}} \psi(r) .
$$

This can be simplified using the Jacobi-Anger identity,

$$
e^{i \boldsymbol{k}_{\perp} \cdot \boldsymbol{r}}=e^{i k_{\perp} r \cos \left(\phi-\phi_{k}\right)}=\sum_{m=-\infty}^{\infty} i^{m} J_{m}\left(k_{\perp} r\right) e^{i m\left(\phi-\phi_{k}\right)},
$$

to obtain

$$
A_{m}\left(\boldsymbol{k}^{\prime}\right)=\int d \boldsymbol{r} \int d k_{z}^{\prime \prime} \int_{0}^{\infty} d k_{\perp}^{\prime \prime} k_{\perp}^{\prime \prime} \sum_{m^{\prime}, m^{\prime \prime}=-\infty}^{\infty}(-i)^{m^{\prime}} V_{m^{\prime \prime}}\left(k_{\perp}^{\prime \prime}, k_{z}^{\prime \prime}\right) J_{m^{\prime \prime}}\left(k_{\perp}^{\prime \prime} r\right) e^{i\left(m^{\prime \prime}+m-m^{\prime}\right) \phi} e^{i\left(k_{z}^{\prime \prime}+k_{z}-k_{z}^{\prime}\right) z} J_{m^{\prime}}\left(k_{\perp}^{\prime} r\right) e^{i m^{\prime} \phi_{k^{\prime}}} \psi(r),
$$


where we omitted a factor $1 / \sqrt{2 \pi}$. Performing the integrals over $\phi$ and $z$ gives us a Kronecker $\delta$ and a Dirac $\delta$ function, which finally gives us for the scattering amplitude

$$
\begin{aligned}
A_{m}\left(\boldsymbol{k}^{\prime}\right) & =\int_{0}^{\infty} d r r \int d k_{z}^{\prime \prime} \int_{0}^{\infty} d k_{\perp}^{\prime \prime} k_{\perp}^{\prime \prime} \sum_{m^{\prime}, m^{\prime \prime}=-\infty}^{\infty}(-i)^{m^{\prime}} V_{m^{\prime \prime}}\left(k_{\perp}^{\prime \prime}, k_{z}^{\prime \prime}\right) J_{m^{\prime \prime}}\left(k_{\perp}^{\prime \prime} r\right) \delta_{m^{\prime \prime}+m-m^{\prime}} \delta\left(k_{z}^{\prime \prime}+k_{z}-k_{z}^{\prime}\right) J_{m^{\prime}}\left(k_{\perp}^{\prime} r\right) e^{i m^{\prime} \phi_{k^{\prime}}} \psi(r) \\
& =\int_{0}^{\infty} d k_{\perp}^{\prime \prime} k_{\perp}^{\prime \prime} \sum_{m^{\prime}=-\infty}^{\infty}(-i)^{m^{\prime}} e^{i m^{\prime} \phi_{k^{\prime}}} V_{m^{\prime}-m}\left(k_{\perp}^{\prime \prime}, k_{z}^{\prime}-k_{z}\right) \int_{0}^{\infty} d r r J_{m^{\prime}-m}\left(k_{\perp}^{\prime \prime} r\right) J_{m^{\prime}}\left(k_{\perp}^{\prime} r\right) \psi(r) .
\end{aligned}
$$

\section{APPENDIX B: VORTEX SCATTERING ON A HELIX, DERIVATION AND PROPERTIES}

Inserting the potential of point scatterers arranged on a helix [Eq. (7)] in the expression for the polar expansion coefficients [Eq. (5)] gives us

$$
V_{m}\left(q_{\perp}, q_{z}\right)=\int d z \int_{0}^{\infty} d r \int_{0}^{2 \pi} d \phi r J_{m}\left(q_{\perp} r\right) e^{-i m \phi} e^{-i q_{z} z} \sum_{j, n=-\infty}^{\infty} \delta(r-R) \delta\left(\phi-a \frac{2 \pi z}{P}+2 \pi n\right) \delta\left(z-\frac{j}{Q} P\right) .
$$

We can rewrite this as

$$
\begin{aligned}
V_{m, v}\left(q_{\perp}\right) & =\frac{1}{P} \int_{0}^{P} d z \int_{0}^{2 \pi} d \phi R J_{m}\left(q_{\perp} R\right) e^{-i m \phi} e^{-i v \frac{2 \pi}{P} z} \sum_{j=1}^{Q} \delta\left(\phi-a \frac{2 \pi z}{P}\right) \delta\left(z-\frac{j}{Q} P\right) \\
& =\frac{R}{P} J_{m}\left(q_{\perp} R\right) \sum_{j=1}^{Q} e^{-i(a m+v) \frac{2 \pi j}{Q}}=\frac{R Q}{P} J_{m}\left(q_{\perp} R\right) \sum_{N} \delta\left(\frac{a m+v}{Q}-N\right),
\end{aligned}
$$

with $v \in \mathbb{Z}$ and $q_{z}=v \frac{2 \pi}{P}$. The latter is quantized because of the periodicity in $z$.

When dropping the constant $\frac{R Q}{P}$, filling the expansion coefficients (B2) into Eq. (A4) gives us for the scattering amplitude

$$
A_{m}\left(\boldsymbol{k}^{\prime}\right)=\int_{0}^{\infty} d k_{\perp}^{\prime \prime} k_{\perp}^{\prime \prime} \sum_{N, m^{\prime}=-\infty}^{\infty}(-i)^{m^{\prime}} e^{i m^{\prime} \phi_{k^{\prime}}} J_{m^{\prime}-m}\left(k_{\perp}^{\prime \prime} R\right) \delta\left(\frac{a\left(m^{\prime}-m\right)+v}{Q}-N\right) \int_{0}^{\infty} d r r J_{m^{\prime}-m}\left(k_{\perp}^{\prime \prime} r\right) J_{m^{\prime}}\left(k_{\perp}^{\prime} r\right) \psi(r),
$$

where the number $v=\frac{P}{2 \pi}\left(k_{z}-k_{z}^{\prime}\right) \in \mathbb{Z}$ discretizes the transferred forward momentum. The integral over $k_{\perp}^{\prime \prime}$ is given analytically by

$$
\int_{0}^{\infty} d k_{\perp}^{\prime \prime} k_{\perp}^{\prime \prime} J_{n}\left(k_{\perp}^{\prime \prime} r\right) J_{n}\left(k_{\perp}^{\prime \prime} R\right)=\frac{\delta(r-R)}{r},
$$

such that we find the final scattering amplitude to be

$$
\begin{aligned}
A_{m}\left(\boldsymbol{k}^{\prime}\right) & =\sum_{N, m^{\prime}=-\infty}^{\infty}(-i)^{m^{\prime}} e^{i m^{\prime} \phi_{k^{\prime}}} \delta\left(\frac{a\left(m^{\prime}-m\right)+v}{Q}-N\right) \int_{0}^{\infty} d r \delta(r-R) J_{m^{\prime}}\left(k_{\perp}^{\prime} r\right) \psi(r) \\
& =e^{i(m-a v) \phi_{k^{\prime}}} \sum_{N=-\infty}^{\infty}(-i)^{a N Q} e^{i a N Q \phi_{k^{\prime}}} J_{m+a(N Q-v)}\left(k_{\perp}^{\prime} R\right) \psi(R)=e^{i(m-a v) \phi_{k^{\prime}}} \sum_{N=-\infty}^{\infty}(-i)^{N Q} e^{i N Q \phi_{k^{\prime}} J_{m-a v+N Q}\left(k_{\perp}^{\prime} R\right) \psi(R) .}
\end{aligned}
$$

When looking at the scattering amplitude for rings for which $v=a m+n Q$ or, equivalently, $m-a v=n Q, n \in \mathbb{Z}$, we get

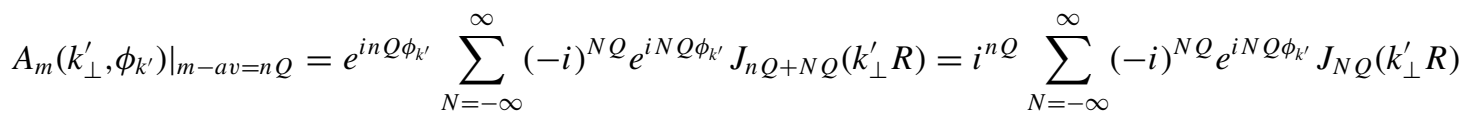

$$
\begin{aligned}
& =J_{0}\left(k_{\perp}^{\prime} R\right)+\sum_{N=1}^{\infty}\left[(-i)^{N Q} e^{i N Q \phi_{k^{\prime}}} J_{N Q}\left(k_{\perp}^{\prime} R\right)+(-i)^{-N Q} e^{-i N Q \phi_{k^{\prime}}} J_{-N Q}\left(k_{\perp}^{\prime} R\right)\right] \\
& =J_{0}\left(k_{\perp}^{\prime} R\right)+\sum_{N=1}^{\infty}(-i)^{N Q} J_{N Q}\left(k_{\perp}^{\prime} R\right)\left(e^{i N Q \phi_{k^{\prime}}}+e^{-i N Q \phi_{k^{\prime}}}\right) \\
& =J_{0}\left(k_{\perp}^{\prime} R\right)+\sum_{N=1}^{\infty} 2(-i)^{N Q} J_{N Q}\left(k_{\perp}^{\prime} R\right) \cos \left(N Q \phi_{k^{\prime}}\right),
\end{aligned}
$$


where we omitted a phase factor $i^{n Q}$. Looking at the scattering amplitude in the point $\left(k_{\perp}^{\prime}, \phi_{k^{\prime}}+\pi\right)$ gives us

$$
\begin{aligned}
\left.A_{m}\left(k_{\perp}^{\prime}, \phi_{k^{\prime}}+\pi\right)\right|_{m-a v=n Q} & =J_{0}\left(k_{\perp}^{\prime} R\right)+\sum_{N=1}^{\infty} 2(-i)^{N Q} J_{N Q}\left(k_{\perp}^{\prime} R\right) \cos \left[N Q\left(\phi_{k^{\prime}}+\pi\right)\right] \\
& =J_{0}\left(k_{\perp}^{\prime} R\right)+\sum_{N=1}^{\infty} 2(i)^{N Q} J_{N Q}\left(k_{\perp}^{\prime} R\right) \cos \left[N Q\left(\phi_{k^{\prime}}\right)\right]=\left.A_{m}^{*}\left(k_{\perp}^{\prime}, \phi_{k^{\prime}}\right)\right|_{m-a v=n Q} .
\end{aligned}
$$

\section{APPENDIX C: EFFECT OF MULTIPLE SCATTERING ON THE SYMMETRY OF HOLZ, DERIVATIONS}

As the crystal potential is periodic, its Fourier transform is a discrete function which is nonzero only at the reciprocal lattice points,

$$
\mathcal{F}[V](\boldsymbol{k})=\sum_{\boldsymbol{g}} V_{\boldsymbol{g}} \delta(\boldsymbol{k}-\boldsymbol{g})
$$

with $\boldsymbol{g}$ being a reciprocal lattice vector. As a consequence, a plane wave propagating through a crystal can scatter only to another wave whose wave vector differs by a reciprocal wave vector, $\boldsymbol{k}^{\prime} \rightarrow \boldsymbol{k}=\boldsymbol{k}^{\prime}+\boldsymbol{g}$. When we take only second-order scattering events between the ZOLZ, FOLZ, and FOLZ* (scattering from FOLZ to ZOLZ for which $\Delta k_{z}<0$ ) into account and neglect higher-order Laue zones contributions, we can write (14) as

$$
\begin{aligned}
& A_{m}^{(2)}(\boldsymbol{k})=\left(\sum_{\boldsymbol{g} \in Z O L Z} V_{\boldsymbol{g}} \delta(\boldsymbol{k}+\boldsymbol{g})+\sum_{\boldsymbol{g} \in F O L Z} V_{\boldsymbol{g}} \delta(\boldsymbol{k}+\boldsymbol{g})+\sum_{\boldsymbol{g} \in F O L Z^{*}} V_{\boldsymbol{g}} \delta(\boldsymbol{k}+\boldsymbol{g})\right) \\
& *\left(\sum_{\boldsymbol{g}^{\prime} \in Z O L Z} V_{\boldsymbol{g}^{\prime}} \delta\left(\boldsymbol{k}+\boldsymbol{g}^{\prime}\right)+\sum_{\boldsymbol{g}^{\prime} \in F O L Z} V_{\boldsymbol{g}^{\prime}} \delta\left(\boldsymbol{k}+\boldsymbol{g}^{\prime}\right)+\sum_{\boldsymbol{g}^{\prime} \in F O L Z^{*}} V_{\boldsymbol{g}^{\prime}} \delta\left(\boldsymbol{k}+\boldsymbol{g}^{\prime}\right)\right) * \mathcal{F}\left[\psi_{m}\right](-\boldsymbol{k}) \\
& =\left[\left(\sum_{\boldsymbol{g} \in Z O L Z} V_{\boldsymbol{g}} \delta(\boldsymbol{k}+\boldsymbol{g}) * \sum_{\boldsymbol{g}^{\prime} \in Z O L Z} V_{\boldsymbol{g}^{\prime}} \delta\left(\boldsymbol{k}+\boldsymbol{g}^{\prime}\right)\right)+\left(\sum_{\boldsymbol{g} \in Z O L Z} V_{\boldsymbol{g}} \delta(\boldsymbol{k}+\boldsymbol{g}) * \sum_{\boldsymbol{g}^{\prime} \in F O L Z} V_{\boldsymbol{g}^{\prime}} \delta\left(\boldsymbol{k}+\boldsymbol{g}^{\prime}\right)\right)\right. \\
& +\left(\sum_{\boldsymbol{g} \in F O L Z} V_{\boldsymbol{g}} \delta(\boldsymbol{k}+\boldsymbol{g}) * \sum_{\boldsymbol{g}^{\prime} \in Z O L Z} V_{\boldsymbol{g}^{\prime}} \delta\left(\boldsymbol{k}+\boldsymbol{g}^{\prime}\right)\right)+\left(\sum_{\boldsymbol{g} \in F O L Z} V_{\boldsymbol{g}} \delta(\boldsymbol{k}+\boldsymbol{g}) * \sum_{\boldsymbol{g}^{\prime} \in F O L Z^{*}} V_{\boldsymbol{g}^{\prime}} \delta\left(\boldsymbol{k}+\boldsymbol{g}^{\prime}\right)\right) \\
& \left.+\left(\sum_{\boldsymbol{g} \in F O L Z^{*}} V_{\boldsymbol{g}} \delta(\boldsymbol{k}+\boldsymbol{g}) * \sum_{\boldsymbol{g}^{\prime} \in F O L Z} V_{\boldsymbol{g}^{\prime}} \delta\left(\boldsymbol{k}+\boldsymbol{g}^{\prime}\right)\right)+\left(\sum_{\boldsymbol{g} \in F O L Z} V_{\boldsymbol{g}} \delta(\boldsymbol{k}+\boldsymbol{g}) * \sum_{\boldsymbol{g}^{\prime} \in F O L Z} V_{\boldsymbol{g}^{\prime}} \delta\left(\boldsymbol{k}+\boldsymbol{g}^{\prime}\right)\right)\right] \\
& \text { * } \mathcal{F}\left[\psi_{m}\right](-\boldsymbol{k}) \text {, }
\end{aligned}
$$

where we dropped the contributions of ZOLZ-FOLZ* and FOLZ*-FOLZ* scattering events since they are energetically forbidden. At this point, we can make a second approximation in which we drop all the terms involving scattering through two reciprocal lattice points in the FOLZ and FOLZ*. These are associated with two high-angle scattering events and therefore are far less likely to occur than the scattering events within the ZOLZ. What remains is

$$
\begin{aligned}
A_{m}^{(2)}(\boldsymbol{k})= & {\left[\left(\sum_{\boldsymbol{g} \in Z O L Z} V_{\boldsymbol{g}} \delta(\boldsymbol{k}+\boldsymbol{g}) * \sum_{\boldsymbol{g}^{\prime} \in Z O L Z} V_{\boldsymbol{g}^{\prime}} \delta\left(\boldsymbol{k}+\boldsymbol{g}^{\prime}\right)\right)+2\left(\sum_{\boldsymbol{g} \in Z O L Z} V_{\boldsymbol{g}} \delta(\boldsymbol{k}+\boldsymbol{g}) * \sum_{\boldsymbol{g}^{\prime} \in F O L Z} V_{\boldsymbol{g}^{\prime}} \delta\left(\boldsymbol{k}+\boldsymbol{g}^{\prime}\right)\right)\right] } \\
& * \mathcal{F}\left[\psi_{m}\right](-\boldsymbol{k}),
\end{aligned}
$$

where we made use of the associativity of the convolution product, $A * B=B * A$. The first term describes two scattering events within the ZOLZ that do not alter the intensity of the FOLZ. So in order to study the symmetry of the FOLZ, we have to look at only the second term.

$$
\left.\left.A_{m}^{(2)}(\boldsymbol{k})\right|_{\boldsymbol{k} \in F O L Z} \propto \sum_{\boldsymbol{g} \in Z O L Z} V_{\boldsymbol{g}} \delta(\boldsymbol{k}+\boldsymbol{g}) * \sum_{\boldsymbol{g}^{\prime} \in F O L Z} V_{\boldsymbol{g}^{\prime}} \delta\left(\boldsymbol{k}+\boldsymbol{g}^{\prime}\right) * \mathcal{F}\left[\psi_{m}\right](-\boldsymbol{k}) \propto \sum_{\boldsymbol{g} \in Z O L Z} V_{\boldsymbol{g}} \delta(\boldsymbol{k}+\boldsymbol{g}) * A_{m}^{(1)}(\boldsymbol{k})\right|_{\boldsymbol{k} \in F O L Z}
$$

where $A_{m}^{(1)}(\boldsymbol{k})$ is the scattering amplitude within the first-order Born approximation of our focused vortex beam from the previous section. Depending on the chirality of the helix and the topological charge of the vortex, we know that this can be a 
centrosymmetric function or not; that is, when we scatter a right-handed vortex on a right handed helix, we get

$$
A_{m}^{(1)}\left(\boldsymbol{k}_{\perp}, \frac{2 \pi}{P}\right)=A_{m}^{*(1)}\left(-\boldsymbol{k}_{\perp}, \frac{2 \pi}{P}\right) .
$$

Also for a crystal with real potential (elastic scattering), we get from Friedel's law

$$
V_{g}=V_{-g}^{*}
$$

The convolution product in Eq. (C4) now yields

$$
\begin{aligned}
\left.A_{m}^{(2)}(\boldsymbol{k})\right|_{\boldsymbol{k} \in F O L Z} & \left.\propto \int d \boldsymbol{k}^{\prime} \sum_{\boldsymbol{g} \in Z O L Z} V_{\boldsymbol{g}} \delta\left(-\boldsymbol{k}-\boldsymbol{k}^{\prime}-\boldsymbol{g}\right) A_{m}^{(1)}\left(\boldsymbol{k}^{\prime}\right)\right|_{\boldsymbol{k}^{\prime} \in F O L Z} \\
& \left.\propto \int d k_{z}^{\prime} \delta\left(k_{z}-k_{z}^{\prime}\right) \int d \boldsymbol{k}_{\perp}^{\prime} \sum_{\boldsymbol{g}_{\perp}} V_{\boldsymbol{g}_{\perp}} \delta\left(-\boldsymbol{k}_{\perp}-\boldsymbol{k}_{\perp}^{\prime}-\boldsymbol{g}_{\perp}\right) A_{m}^{(1)}\left(\boldsymbol{k}_{\perp}^{\prime}, k_{z}^{\prime}\right)\right|_{\boldsymbol{k}^{\prime} \in F O L Z} \\
& \left.\propto \int d k_{z}^{\prime} \delta\left(k_{z}-k_{z}^{\prime}\right) \int d\left(-\boldsymbol{k}_{\perp}^{\prime}\right) \sum_{\boldsymbol{g}_{\perp}} V_{-\boldsymbol{g}_{\perp}} \delta\left(-\boldsymbol{k}_{\perp}+\boldsymbol{k}_{\perp}^{\prime}+\boldsymbol{g}_{\perp}\right) A_{m}^{(1)}\left(-\boldsymbol{k}_{\perp}^{\prime}, k_{z}^{\prime}\right)\right|_{\boldsymbol{k}^{\prime} \in F O L Z} \\
& \left.\propto \int d k_{z}^{\prime} \delta\left(k_{z}-k_{z}^{\prime}\right) \int d \boldsymbol{k}_{\perp}^{\prime} \sum_{g_{\perp}} V_{\boldsymbol{g}_{\perp}}^{*} \delta\left(+\boldsymbol{k}_{\perp}-\boldsymbol{k}_{\perp}^{\prime}-\boldsymbol{g}_{\perp}\right) A_{m}^{*(1)}\left(\boldsymbol{k}_{\perp}^{\prime}, k_{z}^{\prime}\right)\right|_{\boldsymbol{k}^{\prime} \in F O L Z} \\
& \left.\propto A_{m}^{*(2)}\left(-\boldsymbol{k}_{\perp}, k_{z}\right)\right|_{\boldsymbol{k} \in F O L Z}
\end{aligned}
$$

Taking into account double-scattering events that include only one high-angle scattering event between ZOLZ and FOLZ, schematically illustrated in Fig. 1, preserves the centrosymmetry we found before. Moreover, this can easily be extended to dynamical scattering up to any order when neglecting the scattering paths with more than one inter-Laue zone scattering event. Suppose the $n$ th-order Born-approximation for the scattering amplitude $\left.A_{m}^{(n)}(\boldsymbol{k})\right|_{\boldsymbol{k} \in F O L Z}$ is centrosymmetric. The $(n+1)$ th-order is then given by

$$
\left.\left.\left.A_{m}^{(n+1)}(\boldsymbol{k})\right|_{\boldsymbol{k} \in F O L Z} \propto \int d \boldsymbol{k}^{\prime} \sum_{\boldsymbol{g} \in Z O L Z} V_{\boldsymbol{g}} \delta\left(-\boldsymbol{k}-\boldsymbol{k}^{\prime}-\boldsymbol{g}\right) A_{m}^{(n)}\left(\boldsymbol{k}^{\prime}\right)\right|_{\boldsymbol{k}^{\prime} \in F O L Z} \propto A_{m}^{*(n+1)}\left(-\boldsymbol{k}_{\perp}, k_{z}\right)\right|_{\boldsymbol{k} \in F O L Z}
$$

\section{APPENDIX D: WHAT ABOUT OTHER CHIRAL POTENTIALS?}

When one takes a two-dimensional (2D) image of a potential, one can never distinguish between two enantiomorphs since mirroring the crystal along the image plane would change the crystal's potential but not the 2D image. HOLZs in the diffraction pattern, on the other hand, carry information about the crystal in the direction parallel to the direction of view, and one can think of using these to determine the chirality. However, within the kinematical approximation, this is not possible using a conventional probe whose phase is constant in any plane parallel to the image plane.

Take, for instance, a potential $V^{R}(\boldsymbol{r})$ and its enantiomorph $V^{L}(\boldsymbol{r})=V^{R}(x, y,-z)$, obtained by mirroring the potential along the plane of view. Their polar expansion coefficients are related by

$$
\begin{aligned}
V_{m}^{R}\left(k \perp, k_{z}\right) & =\int d z \int_{0}^{\infty} d r \int_{0}^{2 \pi} d \phi r V^{R}(r, \phi, z) J_{m}\left(k_{\perp} r\right) e^{-i m \phi} e^{-i k_{z} z} \\
& =\int d z \int_{0}^{\infty} d r \int_{0}^{2 \pi} d \phi r V^{L}(r, \phi,-z) J_{m}\left(k_{\perp} r\right) e^{-i m \phi} e^{-i k_{z} z} \\
& =\int d z \int_{0}^{\infty} d r \int_{0}^{2 \pi} d \phi r V^{L}(r, \phi, z)(-1)^{m} J_{-m}\left(k_{\perp} r\right) e^{i(-m) \phi} e^{i k_{z} z}=(-1)^{m} V_{-m}^{* L}\left(k_{\perp}, k_{z}\right) .
\end{aligned}
$$

As a consequence, the diffraction patterns $\left|A_{0}\left(\boldsymbol{k}^{\prime}\right)\right|^{2}$ differ by only a rotation of $\pi \mathrm{rad}$.

$$
\begin{aligned}
A_{0}^{R}\left(\boldsymbol{k}^{\prime}\right) & =\int_{0}^{\infty} d k_{\perp}^{\prime \prime} k_{\perp}^{\prime \prime} \sum_{m^{\prime}=-\infty}^{\infty}(-i)^{m^{\prime}} V_{m^{\prime}}^{R}\left(k_{\perp}^{\prime \prime}, k_{z}^{\prime}-k_{z}\right) e^{i m^{\prime} \phi_{k^{\prime}}} \int_{0}^{\infty} d r r J_{m^{\prime}}\left(k_{\perp}^{\prime \prime} r\right) J_{m^{\prime}}\left(k_{\perp}^{\prime} r\right) \psi(r) \\
& =\int_{0}^{\infty} d k_{\perp}^{\prime \prime} k_{\perp}^{\prime \prime} \sum_{m^{\prime}=-\infty}^{\infty}(-i)^{m^{\prime}} V_{-m^{\prime}}^{* L}\left(k_{\perp}^{\prime \prime}, k_{z}^{\prime}-k_{z}\right) e^{i m^{\prime}\left(\phi_{k^{\prime}}+\pi\right)} \int_{0}^{\infty} d r r J_{m^{\prime}}\left(k_{\perp}^{\prime \prime} r\right) J_{m^{\prime}}\left(k_{\perp}^{\prime} r\right) \psi(r) \\
& =\int_{0}^{\infty} d k_{\perp}^{\prime \prime} k_{\perp}^{\prime \prime} \sum_{m^{\prime}=-\infty}^{\infty}(-i)^{-m^{\prime}} V_{m^{\prime}}^{* L}\left(k_{\perp}^{\prime \prime}, k_{z}^{\prime}-k_{z}\right) e^{-i m^{\prime}\left(\phi_{k^{\prime}}+\pi\right)} \int_{0}^{\infty} d r r J_{-m^{\prime}}\left(k_{\perp}^{\prime \prime} r\right) J_{-m^{\prime}}\left(k_{\perp}^{\prime} r\right) \psi(r)=A_{0}^{* L}\left(k_{\perp}^{\prime}, \phi_{k^{\prime}}+\pi, k_{z}^{\prime}\right),
\end{aligned}
$$


where in the next to last step we made use of $J_{m^{\prime}}\left(k_{\perp}^{\prime \prime} r\right) J_{m^{\prime}}\left(k_{\perp}^{\prime} r\right)=(-1)^{m^{\prime}+m^{\prime}} J_{-m^{\prime}}\left(k_{\perp}^{\prime \prime} r\right) J_{-m^{\prime}}\left(k_{\perp}^{\prime} r\right)$ and in the last step we changed $-m^{\prime}$ to $m^{\prime}$ since the summation goes over all possible $m^{\prime}$. The Bessel functions in Eq. (D2) give the same weight to expansion coefficients $V_{m}$ and $V_{-m}$ in the diffraction pattern, and it is this property that makes enantiomorphs indistinguishable using plane waves.

When considering scattering of a vortex beam, on the other hand, in Eq. (A4) we have the term

$$
J_{m^{\prime}+m}\left(k_{\perp}^{\prime \prime} r\right) J_{m^{\prime}}\left(k_{\perp}^{\prime} r\right) \neq J_{-m^{\prime}+m}\left(k_{\perp}^{\prime \prime} r\right) J_{-m^{\prime}}\left(k_{\perp}^{\prime} r\right) .
$$

This term gives a different weight to the contribution of the expansion coefficients $V_{m}$ and $V_{-m}$ in the diffraction pattern, and therefore the diffraction patterns of enantiomorphic potentials in general will look different when using vortex beams even within the kinematical approximation.

As an example we simulated the diffraction pattern of a chiral set of point scatterers with fourfold symmetry and periodicity in the $z$ direction. The beam is pointed exactly on the fourfold rotation axis, making the diffraction pattern fourfold symmetric. Because of the periodicity along the beam direction, the diffraction pattern consists of rings corresponding to ZOLZ and HOLZs. As a basis we took three points with coordinates (in $\AA$ ) $C_{1}=(0.5,0,0), C_{2}=(0.5,0.5,0)$, and $C_{3}=(0.5,0, \pm 0.25)$ for the right-handed and left-handed enantiomorph, respectively. This basis was rotated around the origin over angles $\pi / 2 \mathrm{rad}, \pi \mathrm{rad}$, and $3 \pi / 2 \mathrm{rad}$ and repeated ten times in the $z$ direction with a period of $1 \AA$. These points were considered Huygens point sources emitting waves with a wavelength corresponding to a $300 \mathrm{keV}$ electron beam whose phase depends on the $z$ direction and, in the case
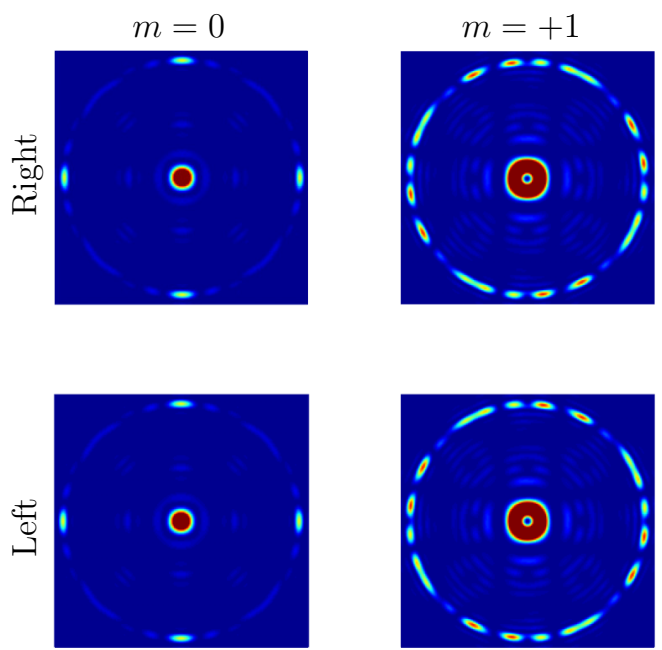

FIG. 7. (Color online) Simulated diffraction pattern of a chiral set of point scatterers with fourfold symmetry for a plane wave and a vortex probe. When the right-handed enantiomorph is mirrored along the beam direction to get the left-handed enantiomorph, the diffraction of the plane wave stays identical, while that of the vortex is different. This shows that vortex beam scattering in the kinematical approximation will, in contrast to plane-wave scattering, depend on the chirality of a potential.

of a vortex probe, on the angular coordinate. The resulting diffraction pattern is shown in Fig. 7. We see that, within the kinematical approximation, the plane-wave diffraction pattern is identical for the left- and right-handed enantiomorphs. When we use a vortex probe, $m=1$, on the other hand, we clearly see a difference between the two diffraction patterns. This demonstrates that vortex beam scattering in general is sensitive to the chirality of a potential, not only to the specific case we investigated in this paper.
[1] P. Goodman and T. Secomb, Acta Crystallogr. A 33, 126 (1977).

[2] P. Goodman and A. Johnson, Acta Crystallogr. A 33, 997 (1977).

[3] G. Friedel, C. R. Acad. Sci. Paris 157, 1533 (1913).

[4] A. Johnson and A. Preston, Ultramicroscopy 55, 348 (1994).

[5] H. Inui, A. Fujii, K. Tanaka, H. Sakamoto, and K. Ishizuka, Acta Crystallogr. B 59, 802 (2003).

[6] J. F. Nye and M. V. Berry, Proc. R. Soc. London, Ser. A 336, 165 (1974).

[7] L. Allen, M. W. Beijersbergen, R. J. C. Spreeuw, and J. P. Woerdman, Phys. Rev. A 45, 8185 (1992).

[8] V. Yu. Bazhenov, M. V. Vasnetsov, and M. S. Soskin, JETP Lett. 52, 429 (1990).

[9] Z.-P. Luo, Y.-L. Sun, and K.-N. An, Appl. Phys. Lett. 76, 1779 (2000).

[10] H. He, M. E. J. Friese, N. R. Heckenberg, and H. RubinszteinDunlop, Phys. Rev. Lett. 75, 826 (1995).

[11] M. E. J. Friese, H. Rubinsztein-Dunlop, J. Gold, P. Hagberg, and D. Hanstorp, Appl. Phys. Lett. 78, 547 (2001).

[12] G. Foo, D. M. Palacios, and G. A. Swartzlander, Opt. Lett. 30, 3308 (2005).

[13] G. A. Swartzlander and R. I. Hernandez-Aranda, Phys. Rev. Lett. 99, 163901 (2007).
[14] E. Serabyn, D. Mawet, and R. Burruss, Nature (London) 464, 1018 (2010).

[15] G. C. G. Berkhout and M. W. Beijersbergen, Phys. Rev. Lett. 101, 100801 (2008).

[16] Structured Light and Its Applications: An Introduction to PhaseStructured Beams and Nanoscale Optical Forces, edited by D. Andrews (Elsevier, Burlington, USA, 2011).

[17] J. Wang, J.-Y. Yang, I. M. Fazal, N. Ahmed, Y. Yan, H. Huang, Y. Ren, Y. Yue, S. Dolinar, M. Tur, and A. E. Willner, Nat. Photonics 6, 488 (2012).

[18] S. Roychowdhury, V. K. Jaiswal, and R. P. Singh, Opt. Commun. 236, 419 (2004).

[19] K. Y. Bliokh, Y. P. Bliokh, S. Savel'ev, and F. Nori, Phys. Rev. Lett. 99, 190404 (2007).

[20] M. Uchida and A. Tonomura, Nature (London) 464, 737 (2010).

[21] J. Verbeeck, H. Tian, and P. Schattschneider, Nature (London) 467, 301 (2010).

[22] A. Béché, R. Van Boxem, G. Van Tendeloo, and J. Verbeeck, Nat. Phys. 10, 26 (2014).

[23] L. Clark, A. Béché, G. Guzzinati, A. Lubk, M. Mazilu, R. Van Boxem, and J. Verbeeck, Phys. Rev. Lett. 111, 064801 (2013). 
[24] J. Verbeeck, H. Tian, and G. Van Tendeloo, Adv. Mater. 25, 1114 (2013).

[25] E. Karimi, L. Marrucci, V. Grillo, and E. Santamato, Phys. Rev. Lett. 108, 044801 (2012).

[26] Z. Mohammadi, C. P. Van Vlack, S. Hughes, J. Bornemann, and R. Gordon, Opt. Express 20, 15024 (2012).

[27] M. D. Graef, Introduction to Conventional Transmission Electron Microscopy (Cambridge University Press, Cambridge, 2003).

[28] Q. Wang, O. Ronneberger, and H. Burkhardt, IIF-LMB, Computer Science Department, University of Freiburg, Technical Report No. 1, 2008 (unpublished).
[29] H. G. Scott, J. Solid State Chem. 66, 171 (1987).

[30] A. Rosenauer and M. Schowalter, in Microscopy of Semiconducting Materials: Proceedings of the 15th Conference, 2-5 April 2007, Cambridge, UK, Springer Proceedings in Physics Vol. 120 (Springer, Dordrect, 2007), p. 169.

[31] C. Ophus, P. Ercius, M. Sarahan, C. Czarnik, and J. Ciston, Acta Crystallogr. A 70, C1455 (2014).

[32] G. Guzzinati, L. Clark, A. Béché, R. Juchtmans, R. Van Boxem, M. Mazilu, and J. Verbeeck, Ultramicroscopy (2014), doi: 10.1016/j.ultramic.2014.10.007.

[33] J. Rusz, J.-C. Idrobo, and S. Bhowmick, Phys. Rev. Lett. 113, 145501 (2014). 